01

\title{
Динамика спектрального состава флуоресценции холодных атомов во внешних электрических и магнитных полях
}

\author{
(C) С.В. Божокин, И.М. Соколов \\ Санкт-Петербургский политехнический университет Петра Великого, \\ 195251 Санкт-Петербург, Россия \\ e-mail: bsvjob@mail.ru, ims@is12093.spb.edu
}

Поступила в редакцию 23.04.2018 г.

На основе последовательного квантового подхода рассчитан сигнал флуоресценции холодного атомного ансамбля, возбуждаемого импульсным излучением и помещенного в постоянное электрическое или магнитное поле. Методами оконного преобразования Фурье проведен анализ спектрального состава этого сигнала и исследовано его изменение с течением времени после окончания возбуждения. Показано, что внешние постоянные поля существенно меняют как форму спектра, так и его динамику. Обнаруженные эффекты объяснены как результат воздействия этих полей на спектр состояний двухатомных квазимолекулярных кластеров, случайно образующихся в неупорядоченных ансамблях.

DOI: $10.21883 /$ OS.2018.09.46542.114-18

\section{Введение}

Атомные ансамбли, приготовленные в специальных ловушках и охлажденные до субдопплеровских температур, обладают целым рядом уникальных физических свойств, что обусловило потенциально широкий круг их применения в задачах стандартизации частоты, квантовой метрологии, квантово-информационных приложениях [1-3].

Эффективность такого применения существенным образом зависит от возможности приготовить используемые атомные ансамбли с требуемыми характеристиками. Во многих случаях важной является возможность управления их свойствами, в частности, оптическими в масштабах реального времени. Один из наиболее простых и эффективных методов такого управления состоит в использовании внешних постоянных электрических или магнитных полей.

Помимо возможности приготовить ансамбли с заданными свойствами, актуальной задачей является разработка эффективных способов экспериментального контроля этих свойств. К настоящему времени одним из самых распространенных методов такого контроля стал анализ флуоресценции этих ансамблей, возбуждаемой либо стационарным, либо импульсным излучением [4-13].

Подобный анализ позволяет также исследовать различные коллективные эффекты, обусловленные обменом фотонами между атомами в условиях больших оптических толщин ансамблей. При исследовании таких процессов, как суб- и суперизлучение, а также слабая и сильная локализация света, очень информативным является анализ динамики затухания интенсивности флуоресценции при возбуждении атомов импульсным излучением. Он позволяет определять времена жизни различных коллективных состояний, так как различные состояния дают вклад на разных интервалах времени после окончания возбуждения. Как показано в работе [14], исследование спектра вторичного излучения и его изменение со временем может дать дополнительную информацию об этих состояниях, а именно об их энергиях.

В настоящей работе мы исследуем изменение спектрального состава флуоресценции и его динамики при наличии упомянутых выше постоянных электрического и магнитных полей, используемых для задач управления.

Как и в работе [14], будем рассматривать случай облаков, приготовленных в магнитооптических ловушках. Основной интерес для нас будет представлять влияние полей на спектр случайно образующихся пар близко расположенных атомов. Такие пары появляются в силу пространственной неупорядоченности этих облаков. Они образуют своего рода квазимолекулы, спектр состояний которых сильно отличается от спектра отдельных атомов и по энергии, и по ширине линий. Их влияние наиболее существенно на временах, больших естественного времени жизни возбужденных состояний свободных атомов, когда другие состояния уже распались.

Вторичное свечение ансамбля является существенно нестационарным процессом. Для анализа спектров таких процессов используются различные подходы $[15,16]$. В работе [15] мы провели сравнение двух основных методов, которые могут быть использованы при исследовании спектров флуоресценции - оконного преобразования Фурье [17,18] и непрерывного вейвлетного преобразования [19-22]. Сравнение показало, что вейвлетное преобразование обладает некоторыми преимуществами. Однако эти преимущества для наших задач не являются принципиальными, и оба подхода позволяют выявить все основные особенности изучаемых сигналов, если правильно подбирать параметры этих преобразований. Поэтому в настоящей работе будем использовать более простой для реализации метод оконного преобразования Фурье. 
Этот подход позволяет организовать вычислительную процедуру таким образом, что спектр вторичного излучения и его изменение со временем вычисляется непосредственно в рамках микроскопического квантового расчета, описывающего динамику рассматриваемой атомно-полевой системы. Конкретные расчеты проведены для различных направлений флуоресценции, а также для различных поляризационных каналов.

\section{Расчет нестационарных сигналов флуоресценции и их спектров}

При расчете спектра флуоресценции, возбуждаемой в импульсном режиме, будем использовать последовательный квантовый подход, разработанный ранее в работе [16]. Не повторяя вывода, отметим лишь его основные особенности. Этот подход базируется на решении нестационарного уравнения Шредингера для волновой функции $\psi$, описывающей состояние объединенной системы, состоящей из всех атомов и электромагнитного излучения.

Рассматриваем неупорядоченный ансамбль, состоящий из $N$ одинаковых атомов, которые считаются неподвижными в силу низкой температуры ансамбля. Медленное тепловое движение, обусловленное конечной величиной температур, и связанные с ним смещения атомов частично учитываются усреднением рассчитываемых величин по их случайному пространственному распределению.

Волновая функция ищется в виде ряда по набору собственных функций гамильтониана невзаимодействующих атомов и поля. Ключевым упрощением в данном подходе является ограничение числа квантово-механических состояний объединенной системы, учитываемых в расчете. Принимая во внимание типичный случай возбуждения слабым излучением, ограничиваемся только состояниями, содержащими не более одного фотона в полевой подсистеме. Как показано в работах $[8,16,23]$, это позволяет корректно описать характер флуоресценции даже с учетом коллективных многоатомных эффектов.

Найденная приближенно волновая функция позволяет анализировать как изменение свойств атомов, так и свойства вторичного излучения, порождаемого в процессе рассеяния. В частности, мы можем рассчитать вектор напряженности вторичного электрического поля $\mathbf{E}(\mathbf{r}, t)$, рассеянного рассматриваемым атомным ансамблем, в произвольной точке, в произвольный момент времени [14]. Знание временной зависимости напряженности дает возможность рассчитать оконное преобразование Фурье для любой поляризационной компоненты $\alpha$ поля. Рассматривая простейший случай прямоугольного окна длительностью $\tau$, середине которого соответствует момент времени $T$, для положительно частотной компонен- ты $E_{\alpha}^{(+)}(\mathbf{r}, \omega, T)$ имеем следующее явное выражение:

$$
\begin{aligned}
E_{\alpha}^{(+)}(\mathbf{r}, \omega, T)=2 \hbar \sum_{n} C_{n}\left(\lambda_{n}\right) \widetilde{\Sigma}_{\alpha}\left(\lambda_{n}\right) \psi_{n} \\
\times \frac{\sin \left(\left(\omega-\lambda_{n}\right) \tau / 2\right)}{\omega-\lambda_{n}} \exp \left(i\left(\omega-\lambda_{n}\right) T\right) .
\end{aligned}
$$

Здесь $\psi_{n}$ и $\lambda_{n}-$ собственные векторы и собственные числа матрицы Грина

$$
\begin{aligned}
G_{e e^{\prime}}(\omega)= & -\left(i \gamma / 2-\omega_{e}\right) \delta_{e e^{\prime}}+\left(1-\delta_{e e^{\prime}}\right) \\
& \times \sum_{\mu, v} \frac{\mathbf{d}_{e g}^{\mu} \mathbf{d}_{g^{\prime} e^{\prime}}^{v}}{\hbar r^{3}} \exp \left(i \frac{\omega_{a} r}{c}\right) \\
& \times\left[\delta_{\mu \nu}\left(1-i \frac{\omega_{a} r}{c}-\left(\frac{\omega_{a} r}{c}\right)^{2}\right)\right. \\
& \left.-\frac{\mathbf{r}_{\mu} \mathbf{r}_{v}}{r^{2}}\left(3-3 i \frac{\omega_{a} r}{c}-\left(\frac{\omega_{a} r}{c}\right)^{2}\right)\right] .
\end{aligned}
$$

Это выражение записано с использованием так называемого полюсного приближения, которое подробно исследовано в работе [24].

В выражении (2) $\mathbf{r}_{\mu}$ - проекция вектора $\mathbf{r}=\mathbf{r}_{a}-\mathbf{r}_{b}$ на оси выбранной системы координат (мы считаем, что в состоянии $\psi_{e^{\prime}}$ возбужден атом $b$, а в состоянии $\psi_{e}-$ атом $\left.a\right), r=|\mathbf{r}|-$ расстояние между атомами $a$ и $b, \mathbf{d}_{e g}$ - матричный элемент оператора дипольного момента, соответствующий переходу атома из состояния $g$ в $e, \omega_{e}$ - частота атомного перехода с учетом штарковского или зеемановского сдвигов, $\omega_{a}-$ частота невозмущенного перехода в отсутствие внешних постоянных полей, $\gamma$ - скорость спонтанного распада возбужденных состояний.

В настоящей работе собственные числа и собственные векторы матрицы (2) определялись численно, как и коэффициенты $C_{n}(\omega)$ разложения по этим векторам вектора $\Lambda_{e}(\omega)$, описывающего возбуждение атомов внешним излучением:

$$
\Lambda_{e}(\omega)=-\frac{\mathbf{d}_{e g} \mathbf{E}(\omega)}{\hbar}=-\frac{\mathbf{u d}_{e g}}{\hbar} E_{0}(\omega) \exp \left(i \mathbf{k r}_{\mathbf{e}}\right) .
$$

Здесь $\mathbf{u}$ - орт поляризации падающего излучения, $E_{0}(\omega)$ - амплитуда его фурье-компоненты.

Вектор $\widetilde{\Sigma}_{\alpha}(\omega)$ в выражении (1), который скалярно умножается на $\psi_{n}$, описывает распространение излучения от атома, возбужденного в состоянии $e$, до точки наблюдения $\mathbf{r}$. В приближении вращающейся волны его явное выражение имеет вид [16]

$$
\begin{aligned}
\widetilde{\Sigma}_{\alpha e}(\omega) & =-\frac{\mathbf{u}_{\alpha}^{\prime *} \mathbf{d}_{g e}}{\hbar r}\left(\frac{\omega}{c}\right)^{2} \exp \left(i \frac{\omega\left|\mathbf{r}-\mathbf{r}_{e}\right|}{c}\right) \\
& =-\frac{\mathbf{u}_{\alpha}^{\prime *} \mathbf{d}_{g e}}{\hbar r}\left(\frac{\omega}{c}\right)^{2} \exp \left(i \frac{\omega r}{c}-i \frac{\mathbf{k}^{\prime} \mathbf{r}_{e}}{c}\right) .
\end{aligned}
$$

Здесь $\mathbf{k}^{\prime}-$ волновой вектор рассеянной волны, $\mathbf{u}_{\alpha}^{\prime}-$ орт поляризации наблюдения. 
Приведенные в этой части работы соотношения позволяют рассчитать динамику спектрального состава излучения, рассеянного в произвольном поляризационном канале и в произвольном направлении.

\section{Результаты}

Полученные в предыдущем разделе общие соотношения могут быть использованы для облаков произвольной формы и с произвольным распределением атомов в них. В этой работе мы рассмотрим сферически симметричные облака со случайным, но однородным в среднем распределением атомов. Будем считать, что атомы имеют основное состояние $J=0$ и возбужденное $J=1$, как это имеет место, например, для атомов стронция. При этом длины входящих в расчет векторов (3), (4), а также $\psi_{n}$ равны $3 N$. Размер матрицы Грина (2) соответственно равен $3 N \times 3 N$. Для описания геометрии рассеяния выбираем систему координат с началом в центре этого облака За направление оси квантования во всех рассмотренных случаях выбираем направление внешнего поля.

Состояние $J=1$, являющееся вырожденным в отсутствие полей, при включении магнитного поля расщепляется на три зеемановских подуровня $-m=-1, m=0$ и $m=1$, имеющих разную энергию. Электрическое поле снимает вырождение частично - подуровни $m=-1$ и $m=1$ имеют одинаковую энергию. Величины внешних полей будем определять по величине вызванного ими расщепления $\Delta$, которое будем измерять в безразмерных единицах, нормируя на величину естественной ширины уровней возбужденного состояния свободного атома. В этой работе ограничимся случаем сильных полей, когда вызванное ими расщепление существенно превышет $\gamma$. Большинство конкретных расчетов проведем для $\Delta=100$. Радиус облака выбираем равным $R=45 \lambda$, а концентрацию $n_{0} \hbar^{3}=0.002$. Здесь и далее в качестве единицы длины используем величину $\lambda$, определяемую волновым числом $\lambda=k^{-1}$ падающего излучения. Единицей времени выберем время жизни возбужденного состояния свободного атома $\tau_{a}=\gamma^{-1}$.

Возбуждающий импульс для простоты считается прямоугольным. Его длительность выбирается много меньше $\tau_{a}\left(\tau_{L} / \tau_{a}=0.1\right)$. Короткие импульсы имеют широкие спектры, что обеспечивает возбуждение большого числа коллективных состояний в широком спектральном интервале. Несущая частота импульса при этом не очень существенна, для определенности выбираем ее равной резонансной частоте того атомного перехода, который возбуждается внешним илучением. В этой работе мы ограничимся двумя основными поляризационными схемами - возбуждение атомов циркулярно поляризованным излучением, волновой вектор $\mathbf{k}$ которого направлен вдоль напряженности постоянного поля, и возбуждение линейно поляризованным светом, распространяющимся перпендикулярно напряженности поля.
Расчет спектра по формулам (1)-(4) показывает, что величина $E_{\alpha}^{(+)}(\mathbf{r}, \omega, T)$ существенно зависит от времени. Помимо зависимости, обусловленной изменением относительной роли различных коллективных состояний, эта величина уменьшается по абсолютной величине изза уменьшения абсолютной величины интенсивности рассеянного излучения. Форма спектра зависит главным образом от плотности атомов. По этой причине далее будем приводить результаты расчета спектров в относительных единицах. Это упрощает процедуру сравнение формы спектров для разных временных интервалов.

Рисунок 1 демонстрирует влияние электрического и магнитного полей на форму спектра вторичного излучения атомного облака, возбуждаемого левоциркулярно поляризованным светом. Для примера приведены результаты расчета циркулярной компоненты флуоресценции на малый угол $\theta=\pi / 20$, причем с сохраняющейся $H \| H$ спиральностью. Отстройка частоты $\delta$ отсчитывается от частоты соответствующего резонансного перехода. Для рассмотренной геометрии это переход $J=0, m=0 \leftrightarrow J=1, m=-1$. Спектры на рис. 1 получены усреднением по конечному числу случайных атомных конфигураций (10000) и не были дополнительно сглажены, поэтому сохраняют следы флуктуаций, связанных со случайным характером пространственного распределения атомов.

Рисунки $1, a$ и $1, b$ соответствуют двум разным моментам времени после окончания импульса возбуждения $\gamma T=50(a)$ и $\gamma T=100(b)$. Видно, что включение электрического или магнитного полей существенно изменяет форму спектра флуоресценции. Для рассмотренной геометрии электрическое поле сохраняет двугорбую форму спектра, уменьшая как расстояние между двумя пиками, так и глубину провала между ними. В магнитном поле имеется один явно выраженный максимум. Помимо формы внешние поля изменяют сдвиг линии атомного перхода. Заметим, что речь не идет о зеемановском или штарковском сдвигах, которые учтены выбором отстройки. Внешние поля модифицируют коллективный лэмбовский сдвиг, обусловленный межатомным взаимодействием [12].

Важным результатом, наблюдаемым на рис. 1, является также влияние полей на динамику спектра. В отсутствие таких полей имеет место существенное уширение спектра со временем и образование провала вблизи резонансной частоты свободного одиночного атома. Это связано с тем, что наиболее долгоживущие коллективные состояния имеют частоты, сильно отличающиеся от частот свободных атомов. Включение полей меняет характер обмена фотонами между атомами и, как следствие, меняет характер коллективных эффектов, уменьшая сдвиги.

Рисунок 1 получен для одного направления и только для канала $H \| H$. Для других направлений и канала с изменяющейся $H \perp H$ спиральностью количественно влияние полей является несколько иным, однако основные качественные особенности, обсуждаемые выше, 
$a$
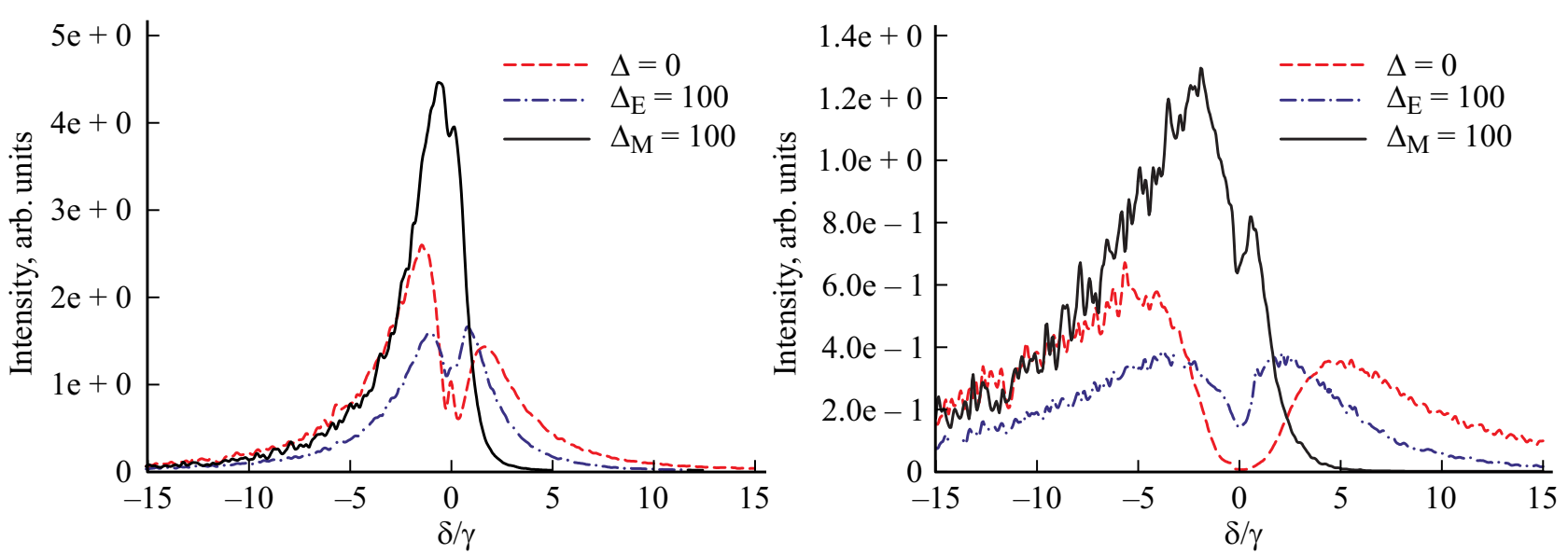

Рис. 1. Спектр излучения флуоресценции для двух моментов времени после окончания импульса возбуждения $\gamma T=50(a)$ и $\gamma T=100(b)$. Поляризационный канал $-H \| H$, возбуждающий импульс резонансен переходу $J=0, m=0 \leftrightarrow J=1, m=-1$, направление наблюдения $\theta=\pi / 20$. Три кривые соответствуют трем случаям - внешние поля отсутствуют, атомы в электрическом поле, атомы в магнитном поле.

сохраняются. Сохраняются они и для второй основной поляризационной схемы, когда линейно поляризованным светом возбуждается так называемый 0-0-переход, т.е. переход $J=0, m=0 \leftrightarrow J=1, m=0$ (рис. 2).

Некоторое отличие этой поляризационной схемы состоит в том, что влияние электрического и магнитного полей оказывается очень близким. Физической причиной этого является то, что оба поля в случае, когда они сильные, делают переход 0-0 фактически изолированным. Фотоны, образующиеся при переходе атомов с уровня $J=1, m=0$ на $J=0, m=0$, не вызывают возбуждений подуровней $J=1, m= \pm 1$. Исключение составляют пары близко расположенных атомов, для которых диполь-дипольное взаимодействие соизмеримо с величиной штарковского или зеемановского расщепления. Именно такие пары обусловливают небольшое отличие сплошных и штрихпунктирных линий на рис. 2.

Изменение динамики отдельных спектральных компонент под действием постоянных полей в широком временном интервале показано на рис. 3. На этом рисунке мы демонстрируем относительный вклад различных компонент, нормируя сигнал на полную интенсивность в каждый момент времени. Это позволяет анализировать спектры, не учитывая быстрое убывание сигнала со временем. Зависимость на рис. 3 показана, начиная с $\gamma T=35$ после окончания импульса возбуждения, что связано с вычислением оконного преобразования Фурье по временному интервалу конечной длины. Выбор интервала, перекрывающегося с этим импульсом, приводит к сильным искажениям получающихся спектров.

Приведенная зависимость позволяет определить, на каких временах состояния с соответствующей энергией дают наиболее существенный вклад в излучение. Для примера на рис. 3 показаны кривые для трех частот: $\delta / \gamma=0$ (рис. $3, a), \delta / \gamma=-10$ (рис. $3, b)$ и $\delta / \gamma=+5$ (рис. 3,c). Видно, например, что сигнал с частотой, совпадающей с частотой атомного перехода (рис. 3,a), быстро затухает после окончания возбуждения, поскольку соответствующие коллективные состояния имеют времена жизни порядка $\tau_{a}$. Наличие поля меняет характер затухания. Особенно ярко проявляется влияние магнитного поля, для которого на резонансной частоте вклад субизлучательных состояний прослеживается на всем приведенном временном интервале. Это связано с упоминавшимся ослаблением лэмбовского сдвига. Долгоживущие коллективные состояния имеют практически ту же частоту, что и короткоживущие.

Отстроенное по частоте излучение дает вклады на бо́льших временах (рис. $3, b$ и 3,c). Для рассмотренного случая хорошо видно немонотонное поведение большинства кривых, которые сначала нарастают, имеют максимум, затем убывают. Положение максимума существенно зависит от наличия поля. Как и на рис. $3, a$ наиболее необычная зависимость наблюдается для флуоресценции в магнитном поле. Для отстройки $\delta / \gamma=-10$ положение максимума соответствует очень большим временам, которые выходят за пределы рассчитанного интервала. Для $\delta / \gamma=+5$, наоборот, максимум имеет место на малых временах. При этом сам сигнал оказывается очень малым по величине.

Наличие сложной временной зависимости и влияние полей на спектры может быть объяснено на основе анализа структуры коллективных состояний. Спектр однократных возбуждений может быть определен на основе расчета собственных чисел $\lambda_{n}$ матрицы (2). Вещественные и мнимые части этих собственных чисел определяют частоты $\omega_{n}=\omega_{a}+\operatorname{Re}\left(\lambda_{n}\right)$ и ширины $\Gamma_{n}=2 \operatorname{Im}\left(\lambda_{n}\right)$ квазимолекулярных состояний [25-27].

При рассматриваемых плотностях основной вклад в послесвечение на больших временах оказывают двух- 
$a$

$b$
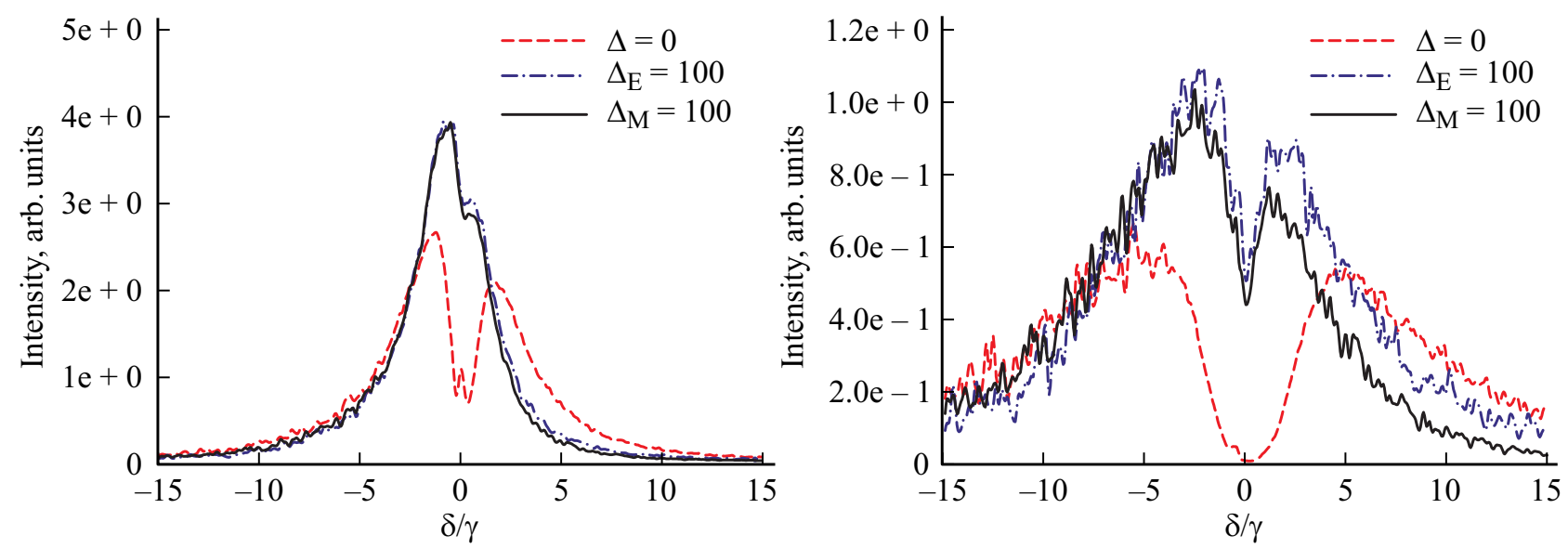

Рис. 2. Спектр излучения флуоресценции для двух моментов времени после окончания импульса возбуждения $\gamma T=50(a)$ и $\gamma T=100(b)$. Поляризационный канал - Lin $\| \operatorname{Lin}$ (сохраняющаяся линейная поляризация), возбуждающий импульс резонансен переходу $J=0, m=0 \leftrightarrow J=1, m=0$, направление наблюдения $\theta=\pi / 2$.

$a$
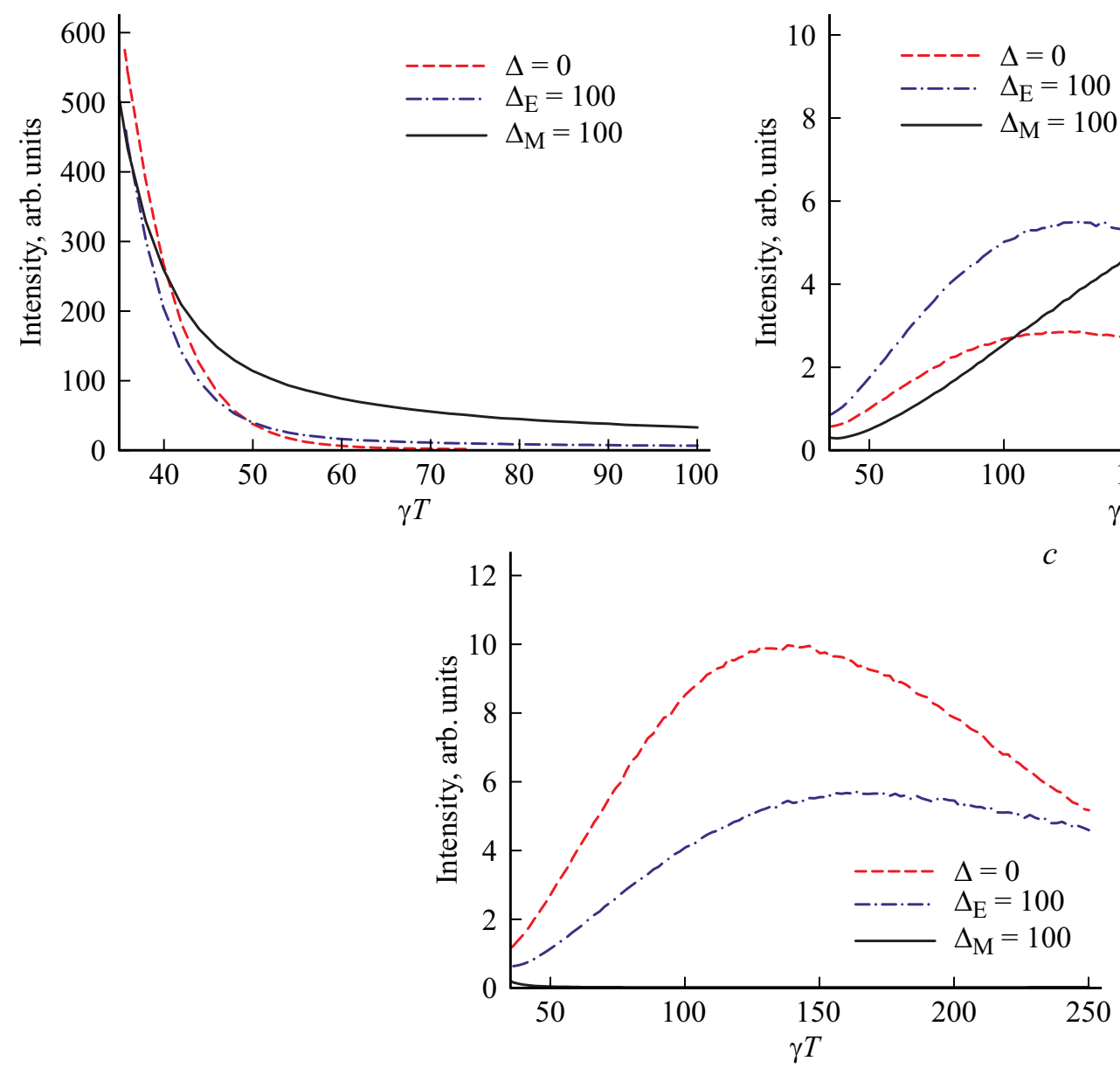

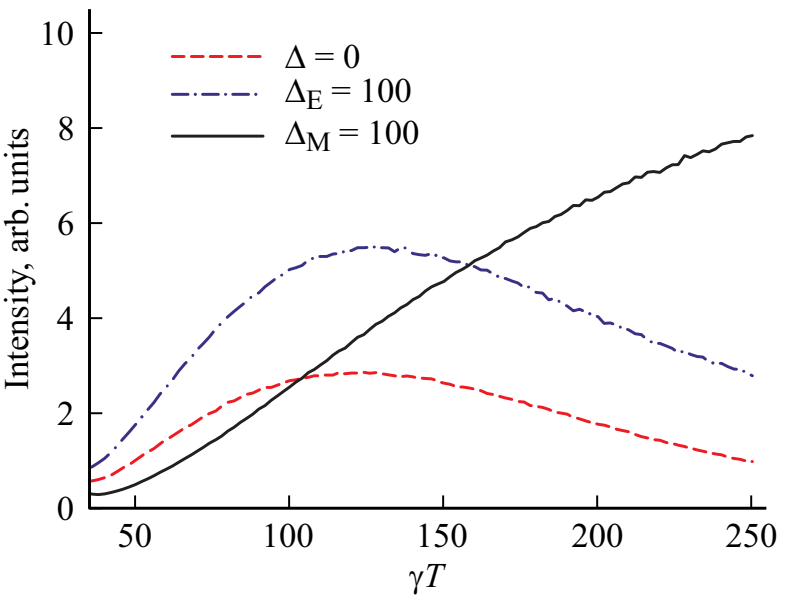

Рис. 3. Величина оконного преобразования Фурье сигнала флуоресценции на угол $\theta=\pi / 20$, соответствующая трем разным частотам: $(a) \delta=0$ - частота равна частоте атомного перехода, $(b) \delta=-10 \gamma-$ частота меньше атомной, $(c) \delta=+5 \gamma-$ частота больше атомной. Расчет проведен для поляризационной схемы $H \| H$. 


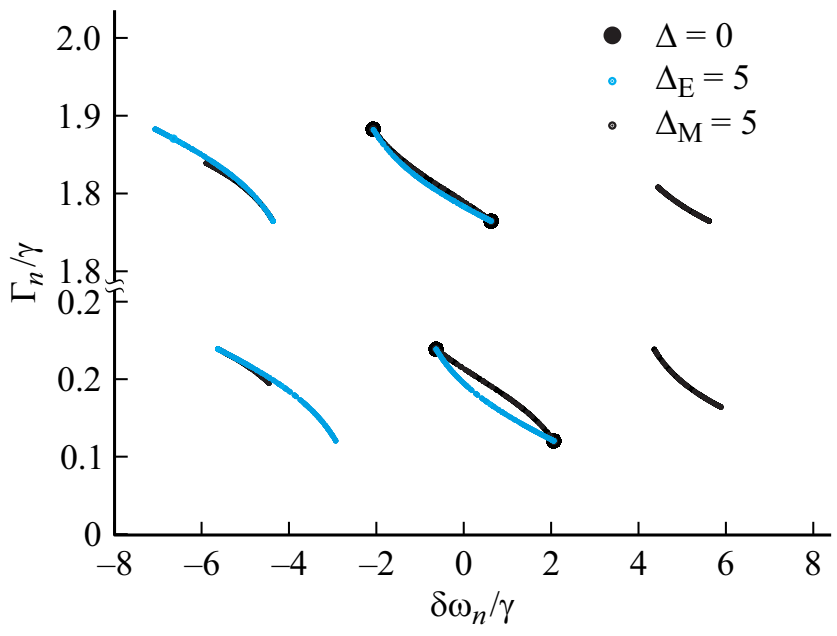

Pис. 4. Спектр однократно возбужденных состояний двухатомной квазимолекулы, расстояние между атомами которой $r_{12}=1.0, \Delta_{\mathrm{E}}=\Delta_{\mathrm{M}}=5$.

атомные кластеры, случайно образующиеся в неупорядоченном ансамбле. Вклады кластеров, содержащих большее число атомов, оказываются несущественными. Для двух атомов с основным состоянием $J=0$ и возбужденным $J=1$ имеются шесть однократно возбужденных состояний. Если атомы расположены далеко друг от друга в свободном пространстве, и их диполь-дипольным взаимодействием можно пренебречь, все шесть состояний имеют одинаковую энергию $E_{e}=\hbar \omega_{a}$ и одинаковую естественную ширину $\gamma$.

При сближении атомов происходит формирование коллективных, квазимолекулярных состояний, обусловленных межатомным взаимодействием. Из шести атомарных состояний формируются шесть молекулярных, три из которых короткоживущие, суперизлучательные, а три - субизлучательные. Две пары состояний имеют одинаковую энергию и одинаковое время жизни. В свободном пространстве в отсутствие внешних полей энергии и времена жизни этих состояний не зависят от ориентации молекулярной оси. Таким образом, на диаграмме „частота уровня-ширина“, имеются четыре различных состояния. На рис. 4 эти состояния отмечены крупными черными точками. При выбранном межатомном расстоянии $r_{12}=1.0$ сдвиги коллективных состояний от частоты атомного перехода изолированного атома $\delta \omega_{n}=\omega_{n}-\omega_{a}$ составляют $\delta \omega_{n} / \gamma \simeq \pm 0.63$ и $\delta \omega_{n} / \gamma \simeq \pm 2.07$, а ширины $-\gamma_{n} / \gamma \simeq 1.9,1.81,0.18,0.096$. Межатомное взаимодействие, таким образом, существенно модифицирует спектр системы.

Помещение взаимодействующих атомов в электрическое или магнитное поля делает систему анизотропной, и энергия каждого из четырех квазимолекулярных состояний начинает зависеть от ориентации молекулярной оси относительно направления напряженности поля. Кроме этого, часть состояний смещается в силу эффекта Штарка или Зеемана. Учитывая это, а также всевозможные ориентации, имеем четыре спектральные полосы в случае электрического поля и шесть для магнитного. Эти полосы показаны на рис. 4 черным и серым цветами. Для компактности рис. 4 построен для случая, когда $\Delta_{\mathrm{E}}=\Delta_{\mathrm{M}}=5$.

Рисунок 4 объясняет также одинаковое влияние разных полей при возбуждении 0-0-перехода. Как видно, вблизи резонансной частоты этого перехода структура уровней в электрическом и магнитном поле близка. При возбуждении перехода $J=0, m=0 \leftrightarrow J=1, m=-1$ влияние полей различно, поскольку ширина полосы состояний в магнитном поле приблизительно в два раза у́же. По этой же причине на рис. 1 для магнитного поля практически отсутствует излучение с положительными отстройками от резонанса.

\section{Заключение}

В представленной работе на основе последовательного квантового микроскопического подхода рассчитан сигнал флуоресценции холодного атомного облака, возбужденного импульсным излучением. Методом оконного преобразования Фурье проведен спектральный анализ этого сигнала. Исследовано влияние постоянного электрического и магнитного полей на характер спектров. Рассмотрены две основные схемы возбуждения - циркулярно поляризованным светом, распространяющимся вдоль направления напряженности поля, и линейно поляризованным светом, волновой вектор которого перпендикулярен вектору напряженности постоянного поля.

Показано, что внешние постоянные поля существенно меняют форму спектра, характер его изменения со временем, а также модифицируют коллективный лэмбовский сдвиг. Обнаруженные эффекты объяснены как результат влияния этих полей на спектр состояний двухатомных квазимолекулярных кластеров, образующихся в неупорядоченных ансамблях.

Все расчеты проведены для холодных атомных облаков, однако подобная методика может быть применена для исследования флуоресценции ансамблей примесных центров в прозрачных диэлектриках $[28,29]$. Анализ спектров их флуоресценции может служить методикой исследования их пространственного распределения. Он позволит определять наличие кластеров, содержащих разное число близко расположенных примесей, что важно для детальной интерпретации прецизионных экспериментов, проводимых с такими ансамблями. Использование внешних постоянных полей может стать эффективным методом управления их оптическими свойствами.

Работа выполнена при финансовой поддержке Российского научного фонда (проект № 17-12-01085). Результаты работы были получены с использованием вычислительных ресурсов суперкомпьютерного центра СанктПетербургского политехнического университета Петра Великого (http://www.spbstu.ru). 


\section{Список литературы}

[1] Hau L.V. // Nature Photonics. 2008. V. 2. P. 451.

[2] Bouwmeester D., Ekert A., Zeilinger A.. The Physics of Quantum Information, Berlin Heidelberg: Springer, 2010.

[3] Bloom B.J, Nicholson T.L., Williams J.R. et al. // Nature. 2014. V. 506. P. 71.

[4] Labeyrie G., Vaujour E., Muller C. A., Delande D., Miniatura C., Wilkowski D., Kaiser R. // Phys. Rev. Lett. 2003. V. 91. P. 223904.

[5] Fioretti A., Molisch A.F., Muller J.H., Verkerk P., Allegrini M. // Opt. Commun. 1998. V. 149. P. 415.

[6] Balik S., Olave R.G., Sukenik C.I., Havey M.D., Datsyuk V.M., Sokolov I.M., Kupriyanov D.V. // Phys. Rev. A. 2005. V. 72. P. 051402.

[7] Balik S., Havey M.D., Sokolov I.M., Kupriyanov D.V. // Phys. Rev. A. 2009. V. 79. P. 033418.

[8] Roof S., Kemp K., Havey M., Sokolov I.M., Kupriyanov D.V. // Optics Letters. 2015. V. 40. P. 1137.

[9] Pellegrino J., Bourgain R., Jennewein S., Sortais Y.R.P., Browaeys A., Jenkins S.D., Ruostekoski J. // Phys. Rev. Lett. 2014. V. 113. P. 133602.

[10] Sokolov I.M., Kuraptsev A.S., Kupriyanov D.V., Havey M.D., Balik S. // J. Mod. Opt. 2013. V. 60. N 1. P. 50.

[11] Araujo M.O., Kresic I., Kaiser R., Guerin W. // Phys. Rev. Lett. 2016. V. 117. P. 073002.

[12] Roof S.J., Kemp K.J., Havey M.D., Sokolov I.M. // Phys. Rev. Lett. 2016. V. 117. P. 073003.

[13] Guerin W., Araujo M.O., Kaiser R. // Phys. Rev. Lett. 2016. V. 116. P. 083601.

[14] Божскин С.В., Соколов И.М. // Опт. и спектр. 2017. Т. 123. N 6. P. 851.

[15] Божскин С.В., Соколов И.М. // ЖТФ. 2018 принята к печати

[16] Соколов И.М., Куприянов Д.В., Хэви М.Д. // ЖЭТФ. 2011. T. 139. C. 288.

[17] Короновский А.А., Макаров В.А., Павлов А.Н. и др. Вейвлеты в нейродинамике и нейрофизиологии. М.: Физматлит, 2013. $273 \mathrm{c}$.

[18] Смоленщев Н.К. Основы теории вейвлетов. Вейвлеты в Matlab. М.: ДНК Пресс, 2008. 448 с.

[19] Mallat S. A Wavelet Tour of Signal Processing. 3rd ed. NY: Academic Press, 2008. 671 p.

[20] Chui C.K. An Introduction to Wavelets. NY.: Academic Press, 1992. $413 \mathrm{p}$.

[21] Bozhokin S.V., Suslova I.B. // Physica A. 2015. V. 421. P. 151.

[22] Божсокин С.В., Жарко С.В., Ларионов Н.В., Литвинов А.Н., Соколов И.М. // ЖТФ. 2017. Т. 87. С. 822.

[23] Kuraptsev A.S., Sokolov I.M. // Phys. Rev. A. 2014. V. 90. P. 012511.

[24] Milonni P.W., Knight P.L. // Phys. Rev. A. 1974. V. 10. P. 1096.

[25] Gero A., Akkermans E. // Phys. Rev. Lett. 2006. V. 96. P. 093601.

[26] Skipetrov S.E., Sokolov I.M., Havey M.D. // Phys. Rev. A. 2016. V. 94. P. 013825.

[27] Sokolov I.M., Kupriyanov D.V., Olave R.G., Havey M.D. // J. Mod. Opt. 2010. V. 57. P. 1833.

[28] Курапцев А.С., Соколов И.М. // ЖЭТФ. 2016. Т. 150. № 2(8). C. 275.

[29] Kuraptsev A.S., Sokolov I.M. // Phys. Rev. A. 2016. V. 94. P. 022511. 